\section{Smoking delays the response to treatment in episcleritis and scleritis}

ZFHM Boonman' ${ }^{1}$, RJW de Keizer ${ }^{1}$ and PG Watson ${ }^{1,2,3}$

\begin{abstract}
Purpose To evaluate the influence of smoking on comorbidity, treatment, visual and general outcome in patients with scleritis. Methods The smoking habits of 103 patients with a diagnosis of episcleritis or scleritis were evaluated. These patients were treated by one ruling protocol at the Leiden University Medical Center between 1997 and 2000.

Medical records of each patient were evaluated in detail. Data on possible factors concerning smoking were collected by postal questionnaire.
\end{abstract}

Results Of all 103 patients diagnosed with either episcleritis or scleritis, $41(39.8 \%)$ were smoking during treatment of the scleral inflammation. In total, 19 patients $(18.4 \%)$ had a smoking history while $43(41.7 \%)$ patients have never smoked. The response to any of the given medications could be delayed by at least 4 weeks in many smoking patients (odds ratio (OR) 5.4 [95\% confidence interval 1.9-15.5]), particularly those with posterior scleritis. Smoking patients above the age of 48 years were even more likely to respond belatedly to any given therapy (OR 6.6 [2.1-20.7]). However, having a smoking history did not delay the response. Furthermore, smoking did not worsen the visual prognosis and was not associated with additional recurrences or ocular complications after successful treatment.

Conclusions Although scleritis patients who smoked during treatment eventually responded, there was frequently over a month's delay before the medication became effective when compared to nonsmokers. This was irrespective of the type of disease or given therapy. As a consequence, smokers required more intensive therapy than those who did not smoke.
Eye (2005) 19, 949-955. doi:10.1038/sj.eye.6701731; published online 5 November 2004

Keywords: scleritis; episcleritis; smoking; treatment

\section{Introduction}

This study was instituted to determine the effect of smoking on the treatment of smoking patients with episcleritis or scleritis compared to nonsmoking patients. We studied whether smoking scleritis patients had more ocular complications, more severe disease or needed longer and more intensive treatment than nonsmokers and whether smoking influenced the visual prognosis and outcome after treatment.

Inflammation of the wall of the eye and its mesodermal coverings can present in many ways from benign simple episcleritis to the severe necrotizing scleritis. The differential diagnosis of episcleritis from scleritis and between diffuse, nodular, and necrotizing disease affecting the anterior segment of the eye is well-recognised. ${ }^{1}$ It is also known that the condition with which the patient first presents usually continues throughout the course of the disease. ${ }^{2}$ The treatments given follow a wellrecognised protocol depending on the ocular and systemic condition of the patients (Table 1$){ }^{3}$ Those with diffuse and nodular scleritis are treated initially with nonsteroidal antiinflammatory medication (NSAIDs). Oral steroids in immunosuppressive doses are given to recalcitrant cases and those in whom vision is threatened, particularly patients with posterior scleritis and those with necrotizing scleritis. Disease-modifying agents (DMARDs) are added in those with other systemic diseases, especially if they have a systemic vasculitis or they fail to respond to therapy. Intravenous
${ }^{1}$ Department of Ophthalmology, Leiden University Medical Center, Leiden,

The Netherlands

${ }^{2}$ Department of Clinical Ophthalmology, Institute of Ophthalmology and Moorfields Eye Hospital, London, UK

${ }^{3}$ Addenbrookes Hospital, Cambridge, UK

Correspondence: ZFHM Boonman, Department of Ophthalmology (J3-S), Leiden University Medical Center, Albinusdreef 2 PO Box 9600,2300 RC, Leiden, The Netherlands Tel: ++ 31715263 938; Fax: ++31715262888 E-mail: Z.F.H.M.Boonman@ LUMC.nl

Received: 13 April 2004 Accepted in revised form: 2 September 2004 Published online:

5 November 2004

Data in this paper have not been presented in any meeting.

Financial support: None. Commercial interest: None. 
Table 1 Treatment modalities in patients with episcleritis or scleritis

\begin{tabular}{lllll}
\hline & & Treatment modality: from left to right in increasing order of intensity & \\
\hline Episcleritis & Artificial tears & Topical NSAID drops & $\begin{array}{l}\text { Mild steroid drops } \\
\text { (eg Fluorometholone) }\end{array}$ & $\begin{array}{l}\text { Oral NSAIDs and/ } \\
\text { or topical NSAIDs }\end{array}$ \\
Scleritis & $\begin{array}{l}\text { Oral NSAIDs and/or } \\
\text { topical NSAIDs }\end{array}$ & $\begin{array}{l}\text { Oral steroids and/or } \\
\text { topical steroids }\end{array}$ & $\begin{array}{l}\text { (eg cyclophosphamide, methotrexate, } \\
\text { cyclosporine, azathioprine }\end{array}$ & \\
\hline
\end{tabular}

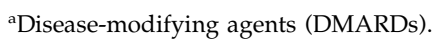

therapy is used in those with acute sight-threatening disease or exceptionally severe disease.

Smoking can not only induce diseases but also affects drug interactions that are clinically relevant during treatment. ${ }^{4}$ According to the World Health Organization, the Netherlands has one of the highest percentage rates of smoking in the EU. In year 2002, 30.1\% of the Dutch population older than 15 years smoked. Another $30 \%$ of the Dutch inhabitants are ex-smokers. ${ }^{5}$ These high percentages make the awareness of possible interactions between smoking and various drug treatments of daily practical importance.

Most studies have been conducted to investigate which systemic diseases are induced by long-term smoking habits. ${ }^{6-9}$ Fewer studies have been looking into the effect of smoking on the severity of the disease itself ${ }^{10-12}$ or the effect it has on its treatment. ${ }^{13,14}$ This study addresses these issues.

\section{Patients and methods}

We determined the smoking habits and reviewed the records of 132 patients with episcleritis or scleritis attending the department of Ophthalmology at the Leiden University Medical Center between January 1997 and April 2000. The treatment regimens were prescribed by one of us (RdeK) in a general episcleritis/scleritis protocol. Of the 132 records identified, 29 were excluded because of insufficient clinical detail to ensure a correct diagnosis or lack of insufficient follow-up data for the 3 -year study period. The follow-up period from the start of treatment was at least 6 months. All the patients were examined at regular intervals according to the severity of the disease during the 3 years prior to the end of the study. Data on possible factors concerning smoking were collected by postal questionnaire. Information about smoking habits was obtained after determining the patient's response to treatment. Patients who did not return the survey were telephoned personally and asked about their smoking habits. When a patient had died, information about tobacco usage was obtained through patients' partners, family or their general practitioners.

Scleritis was divided into anterior and posterior. Anterior scleritis incorporated diffuse, nodular, necrotizing with inflammation (necrotizing), and necrotizing without inflammation (scleromalacia perforans). Episcleritis was not further subclassified into diffuse or nodular. Posterior scleritis was diagnosed clinically and by a B scan ultrasonograph using the Biovision-B-S with a $10 \mathrm{MHz}$ transducer plus a standardized A-mode transducer and techniques of standardized ultrasonography.

All the patients were examined by an internist, rheumatologist, otorhinolaryngologist or neurologist. The visual acuity was measured at presentation and at the end of follow-up. Visual loss was considered to be defective if there was a reduction of two Snellen lines or more. Another disease or complication that may have caused a reduction in visual acuity was recorded to determine that the visual loss could be solely attributed to the scleral inflammation. The data concerning the patients were entered into a computerized database to ensure that it was retrievable.

An immediate good response to treatment was defined as relief from pain and headache, and resolution of redness and oedema of the episcleritis or scleritis. Treatment varied according to the underlying condition but effective treatment should relieve pain within a few hours. Resolution of the physical signs is generally slower depending on the type of disease but they should also resolve within a month. A delayed response was defined as failure of resolution of signs and symptoms after 4 weeks. Resolution either before or after the 4 weeks cutoff was entered as a binary dependent variable in a forward logistic regression model.

Complete remission was considered to have happened if no signs or symptoms remained at the end of the course of treatment. Remission with complications was used if signs and symptoms had disappeared but the patient was left with poor vision, residual signs in the cornea, uvea, lens, or retina or if there was scleral tissue loss. Visual loss, corneal changes, and uveitis can occur in all forms of episcleritis and scleritis

Forward stepwise logistic regression analysis was undertaken to determine whether smoking or other recorded variables had predictive value for responsiveness to treatment. Outcome in terms of visual loss and cure of episcleritis or scleritis with or without 
complications, and recurrences were also analysed in a similar manner. The SPSS statistical software version 10 (SPSS inc.) was used for statistical analyses.

\section{Results}

Smoking information was gathered from all $(N=103)$ of those treated for scleritis. Smoking tobacco was used by $41(39.8 \%)$ out of 103 patients during the period of diagnosis or treatment for scleritis or episcleritis. Another 19 (18.4\%) out of 103 patients had a smoking history. Only 43 (41.7\%) out of 103 patients had never smoked. Most smoking scleritis patients were smoking cigarettes and some of them smoked cigars or pipe often in addition to cigarette smoking. These data were obtained during clinic attendances and by a postal questionnaire to which $90(87.4 \%)$ out of 103 patients had replied. Missing information from the remainder was gathered by personally questioning the patients or close relatives of these patients on the telephone.

Of the 103 patients, $76(73.8 \%)$ had scleritis and 27 (26.2\%) had episcleritis. Of the 41 smokers, 13 (31.7\%) had episcleritis, six (14.6\%) had diffuse scleritis, three $(7.3 \%)$ had nodular scleritis, another three $(7.3 \%)$ had necrotizing scleritis, and $16(39 \%)$ had posterior scleritis. Out of 103 patients, 21 (20.4\%) female and 20 (19.4\%) male patients smoked. Their ages were from 17 to 86 years with a mean age of 47.3 years (SD 17.9 years), which is comparable to the nonsmoking group with a minimum age of 21 years, a maximum of 85 and a mean age of 48.3 years (SD 15.2 years) (Table 2 ).

The visual acuity was measured at presentation and at the end of follow-up. Smokers did not have an increased risk of developing poor vision before or after treatment when compared to the nonsmoking patients. Of the 41 smoking patients, $11(26.8 \%)$ had visual loss at presentation of whom seven $(17.1 \%)$ still had reduced vision after treatment. However, having an associated eye disease other than scleritis, such as uveitis, glaucoma, or a developing cataract influences the visual prognosis negatively (odds ratio $\left(\mathrm{OR}_{\mathrm{vision}}\right.$ at diagnosis $) 4.8[95 \%$ confidence interval 1.8-13.5] and $\mathrm{OR}_{\text {vision after treatment }}$ 9.2 [3.0-28.7]). If a smoking scleritis patient has a concomitant eye disease, his chances of having bad vision at presentation are somewhat increased $\left(\mathrm{OR}_{\mathrm{vision}}\right.$ at diagnosis 5.1 [1.8-13.7]), although no added risk exists after treatment of scleritis $\left(\mathrm{OR}_{\mathrm{vision}}\right.$ after treatment 9.2 [3.0-28.7]) compared to the nonsmoking group.

Ocular complications (Table 2: cornea abnormalities and associated eye diseases) developing during the course of the disease consisted of cornea and/or scleral melting, cataract, keratitis, high intraocular pressure, glaucoma, and uveitis. These occurred in 18 (43.9\%) out of 41 smoking patients and in $22(35.5 \%)$ out of 62 nonsmoking patients. There was no statistically significant difference between the two groups. Patients were treated both for their scleritis and additional ocular complication. The additional eye disease responded completely in most cases after treatment.

Associated systemic diseases were almost evenly distributed: $11(26.8 \%)$ out of 41 in the smoking and 18 $(29.1 \%)$ out of 62 in the nonsmoking group. Associated systemic diseases consisted of associated connective tissue - or vasculitis diseases, such as rheumatic arthritis, Sjogren's syndrome, Wegener's granulomatosis, periarteritis nodosa, systemic vasculitis, and systemic lupus erythematosus.

The response to treatment in smoking scleritis patients as measured by reduction or elimination of pain, reduction or resolution of inflammation, or healing of necrotic tissue can easily be determined by direct observation. Resolution was considerably slower in the smoking patients when compared to the nonsmoking patients (OR 5.4 [1.9-15.5]). An effective response to any of the prescribed medication against scleritis was delayed by at least 4 weeks or more in many smoking patients. This was particularly apparent in smoking patients with posterior scleritis in whom $10(62.5 \%)$ out of 16 patients responded belatedly as compared to only two (10\%) out of 20 nonsmoking patients with posterior scleritis (Table 2). The majority, 56 (90.3\%) out of 62 nonsmoking patients responded to the first given therapy compared to only 26 out of $41(63.4 \%)$ smoking patients. However, patients with a smoking history responded in a similar manner to treatment as nonsmoking patients. Being a heavy ( $\geqslant 20$ cigarettes), intermediate (10-20 cigarettes), or light smoker $(\leqslant 10$ cigarettes) also did not make a difference in the response to treatment. Patients with bilateral disease responded in a similar manner to those with unilateral disease.

Being an older smoker is an important risk factor. Older smokers ( $\geqslant 48$ years) had an increased risk of responding slowly to therapy than young smokers (OR 6.6 [2.1-20.7]). Smoking patients with additional associated eye diseases or corneal problems also responded slowly to treatment when compared to nonsmoking patients with similar concomitant eye and cornea diseases (OR 6.2 [2.0-18.9]). In addition, smoking patients with an associated connective tissue - or vasculitis disease reacted more slowly to a medication than nonsmoking patients with a similar systemic disease (OR 5.5 [1.9-15.8]). Importantly, when a smoking patient had all above-mentioned risk factors ( $\geqslant 48$ years with a concomitant eye disease and connective tissue and/or vasculitic disease), the risk of delayed response to initial given treatment was 7 times higher than in a nonsmoking patient with similar risk parameters (OR 6.9 [2.0-24.8]) 
Table 2 Characteristics of smoking and nonsmoking patients with episcleritis/scleritis

\begin{tabular}{|c|c|c|c|c|c|c|c|c|c|c|c|c|}
\hline \multirow[t]{3}{*}{ Characteristics } & \multicolumn{6}{|c|}{ Nonsmoking patients } & \multicolumn{6}{|c|}{ Smoking patients } \\
\hline & \multirow[t]{2}{*}{ Episcleritis } & \multicolumn{4}{|c|}{ Scleritis } & \multirow[t]{2}{*}{ Total } & \multirow[t]{2}{*}{ Episcleritis } & \multicolumn{4}{|c|}{ Scleritis } & \multirow[t]{2}{*}{ Total } \\
\hline & & $\begin{array}{l}\text { Diffuse } \\
\text { anterior }\end{array}$ & $\begin{array}{l}\text { Nodular } \\
\text { anterior }\end{array}$ & Necrotizing & Posterior & & & $\begin{array}{l}\text { Diffuse } \\
\text { anterior }\end{array}$ & $\begin{array}{l}\text { Nodular } \\
\text { anterior }\end{array}$ & Necrotizing & Posterior & \\
\hline Number of patients & 14 & 13 & 13 & 2 & 20 & 62 & 13 & 6 & 3 & 3 & 16 & 41 \\
\hline \multicolumn{13}{|l|}{ General features } \\
\hline Age (SD) & 41.9 (15.4) & $40.2(10.5)$ & $53.6(14.1)$ & $54.0(22.6)$ & $53.9(15.1)$ & $48.3(15.2)$ & $43.5(11.2)$ & $47.5(21.1)$ & $40.0(18.5)$ & $37.3(4.2)$ & $53.6(21.4)$ & $47.3(17.7)$ \\
\hline Women & 11 & 10 & 11 & 2 & 13 & 47 & 7 & 4 & 2 & 1 & 7 & 21 \\
\hline Age of women (SD) & 41.9 (16.6) & $40.3(9.4)$ & $53.6(12.4)$ & $54.0(22.6)$ & $54.8(16.3)$ & $48.4(15.3)$ & $42.9(12.5)$ & $48.0(12.6)$ & $30.5(12.0)$ & 36.0 & $57.3(25.9)$ & $47.1(18.9)$ \\
\hline Men & 3 & 3 & 2 & - & 7 & 15 & 6 & 2 & 1 & 2 & 9 & 20 \\
\hline Age of men (SD) & $41.7(12.5)$ & $40.0(16.1)$ & $53.5(28.9)$ & - & $52.4(13.9)$ & 47.9 (15.5) & $44.2(10.6)$ & $46.5(41.7)$ & 59.0 & $38.0(5.7)$ & $50.7(18.1)$ & $47.5(16.9)$ \\
\hline Bilateral & 1 & 4 & 4 & 1 & 8 & 18 & 1 & 3 & 2 & 2 & 4 & 12 \\
\hline \multicolumn{13}{|l|}{ Complications } \\
\hline Visual loss at presentation & 1 & 1 & 5 & 1 & 7 & 15 & 1 & 1 & - & 2 & 7 & 11 \\
\hline Cornea abnormalities & 2 & - & 3 & 1 & 2 & 8 & 1 & 2 & 1 & - & 2 & 6 \\
\hline Associated eye diseases & 2 & 1 & 2 & 1 & 8 & 14 & 1 & 1 & 1 & - & 9 & 12 \\
\hline Associated systemic diseases ${ }^{a}$ & 2 & 6 & 1 & 2 & 7 & 18 & 1 & 2 & 2 & 3 & 3 & 11 \\
\hline \multicolumn{13}{|l|}{ Responsiveness to treatment } \\
\hline Delayed response ${ }^{\mathrm{b}}$ & - & 1 & 2 & 1 & 2 & $6(9.7 \%)$ & 2 & - & 2 & 1 & 10 & $15(36.6 \%)$ \\
\hline Immediate response & 14 & 12 & 11 & 1 & 18 & $56(90.3 \%)$ & 11 & 6 & 1 & 2 & 6 & $26(63.4 \%)$ \\
\hline \multicolumn{13}{|l|}{ Outcome } \\
\hline Complete remission & 11 & 8 & 8 & 1 & 13 & 41 & 10 & 6 & 1 & 2 & 8 & 27 \\
\hline Remission with complications & 3 & 4 & 4 & 1 & 7 & 19 & 2 & - & 2 & 1 & 7 & 12 \\
\hline No remission & - & - & - & - & - & - & - & - & - & - & 1 & 1 \\
\hline Visual loss after treatment & - & - & 5 & 1 & 5 & 11 & - & - & - & - & 7 & 7 \\
\hline
\end{tabular}

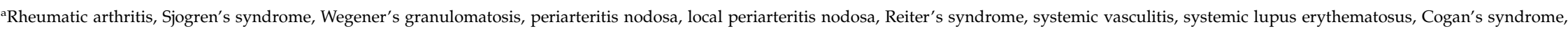
ankylosing spondylitis, Crohn's disease, sarcoidosis, thyrotoxicosis, rhinosporidiosis.

'Minimum delay of 4 weeks. 
The general outcome after treatment was not worsened in smoking patients as compared to nonsmoking patients, although one smoking patient failed to respond to treatment at all and the eye had to be enucleated. All the other smoking and nonsmoking patients went into complete remission from episcleritis or scleritis after treatment. In addition, smoking patients did not develop more recurrences or ocular complications after remission than nonsmoking patients. Conversely, smoking did influence the general outcome indirectly. Patients with risk factors such as, associated systemic diseases (OR 3.5 [1.4-8.9]) and associated eye diseases (OR 4.7 [1.8-12.8]) have an already higher risk of a worse outcome than patients without these risk factors $\left(\mathrm{OR}_{\text {associated systemic diseases \& eye diseases }} 6.6\right.$ [2.2-20.1]). If a patient smokes besides these problems, then there is a slightly increased risk of getting a worse outcome in contrast to a nonsmoking patient with similar risk factors (OR 7.0 [2.2-21.5]).

\section{Discussion}

This study shows that irrespective of the type of medication smoking during treatment delays the full response to the therapy by a month or more in many patients with episcleritis or scleritis when compared to nonsmokers. Out of 62 nonsmokers $56(90.3 \%)$ responded to their first given medication as opposed to only 26 $(64 \%)$ of 41 smoking patients. The delay in response was particularly noticeable in $10(62.5 \%)$ out of the 16 smoking patients with posterior scleritis as compared to only two $(10 \%)$ out of 20 nonsmoking patients with posterior scleritis. In addition, posterior scleritis alone does not increase the risk of responding belated to therapy (not statistically significant). Thus, smoking is the risk factor and not posterior scleritis itself. Practically, this means that smoking patients receive medicine for longer time periods, often at a higher dosage and/or requiring additional stronger treatment.

Episcleritis and scleritis are almost unique in being conditions where it is possible to monitor the effect of treatment by direct observation of the inflammatory response. These observations can also be confirmed by the use of indocyanin green (ICG) and fluorescein angiography. ${ }^{15}$ It has therefore been possible to assess with confidence the effect of smoking on the various methods of treating these conditions. Treatments, given under the supervision of one person, were appropriate for the condition concerned. They varied from a short course of NSAIDs to intensive intravenous steroid and DMARD therapy. The response to treatment was judged by the relief of pain and tenderness and the objective observation of the changes in the external and inner eye supported where necessary by angiography.
A patient who has not been smoking more than a year prior to treatment responds in a similar way to a prescribed treatment when compared to patients who have never lit a cigarette in their life. Thus, previous exposure to tobacco did not have an effect on treatment response. Additionally, there was no difference in response to treatment between heavy ( $\geqslant 20$ cigarettes) and light smokers ( $\leqslant 10$ cigarettes). This suggests that the influences of smoking on belated response to treatment are due to pharmacological interactions or immunopathogenic mechanisms as opposed to the cumulative damage seen, for example, in atherosclerosis or chronic obstructive airway diseases, which makes these diseases more difficult to treat even when a patient has given up smoking. As eventually, smokers pass into complete remission from the scleral inflammation with similar ocular complications, recurrences, and visual outcome; when compared to the results in the nonsmoking patients, it seems that smoking directly influences the effectiveness of specified medication.

The most important pharmacological active components in tobacco smoke are polycyclic aromatic carboxides and nicotine. Other components are carbon monoxide, ammonia, acrolein, acetone, benzopyrenes, hydroquinone, nitrogen oxides, and cyanide. Only few of the many substances in tobacco smoke have been directly investigated for their effect on drugs and it remains unclear as to which specific product is responsible for any changes observed. However, the interaction between the prescribed medicine and smoking have been conducted into the treatment of several diseases, such as in Graves' ophthalmopathy in which smoking during various therapies decreases the efficacy of the given treatment. ${ }^{12-14}$ It is recognized that smoking lowers serum levels of some medications, such as theophyllin, ${ }^{16}$ tricyclic antidepressants, ${ }^{17}$ selective serotonin-reuptake inhibitors, ${ }^{18}$ and antipsychotics ${ }^{19,20}$ mostly because of elevated enzyme induction in the liver, indicating an increased metabolism and therefore a more rapid biotransformation of medicine. ${ }^{21}$ Therefore, smokers need higher dosage of medicine in order to have proper serum levels for similar therapeutic effect when compared to nonsmokers. In addition, older smokers also respond more slowly to treatment than young smokers. As people grow old, various physiological mechanisms react less rapidly, particularly liver metabolism, because of smaller liver size and reduced blood flow in older patients. Although these mechanisms counteract the main smoking effect, ${ }^{22}$ older smokers $(\geqslant 48$ years of age) have a higher number of smoking years than younger smokers and thus have a higher chance of accumulative damage to vulnerable organs and systems. 
Smoking is known to influence the effect or side effect of given medications (pharmacodynamic interaction). This is known to happen in smoking patients with diabetes mellitus type 1 who use significantly higher amounts of insulin than nonsmoking diabetes patients. ${ }^{23}$ Some medications are known to be not as effective in smoking patients such benzodiazepines, ${ }^{24}$ the opioid analgesic (Dextropropoxyfen), ${ }^{25}$ and cardiovascular medication such as the $\beta$-blocker propranolol. ${ }^{26,27}$

There are multiple effects of smoking on immune and inflammatory functions, including increased numbers of circulating and tissue neutrophils and lymphocytes and complex alterations of macrophage, B- and T-cell functional responses, depressed natural killer cell function, and increased complement activation. ${ }^{28-30}$ Smoking can exacerbate inflammatory responses, such as in periodontal disease ${ }^{31}$ and rheumatoid arthritis (RA). ${ }^{32}$ Additionally, refraining from smoking prevents the development or worsening of Graves' ophthalmopathy. ${ }^{12}$ However, contrarily, cessation of cigarette smoking activates the mucocutaneous symptoms, especially oral aphthous lesions in patients with Behcet's disease. ${ }^{33}$ These effects of smoking in inflammatory diseases can be due to an immunological shift from Th1 to Th2 cells in smokers, which gives an immunosuppressive but eventually damaging result. ${ }^{34}$ Smoking can be associated with development of systemic autoimmune disease, including Goodpasture's syndrome, ${ }^{8} \mathrm{RA},{ }^{35}$ Crohn's disease, ${ }^{9} \mathrm{MS},{ }^{7}$ and Grave's disease. ${ }^{11}$ Furthermore, smoking can displace the phenotype of chronic irritable bowel disease from ulcerative colitis to Crohn's disease, regulating not just whether there is a disease but also the type of disease that develops. ${ }^{9}$

There is no evidence that scleritis, which is often linked with an autoimmune disease, is associated with smoking, but this study shows that the speed of response to treatment is reduced and there is the increased chance of slower recovery in many smoking patients. Smoking scleritis patients with additional ocular complications and associated systemic diseases react even slower to the given treatment in contrast to nonsmoking patients with similar severe disease. These patients also have a worse general outcome. The symptoms, ocular complications, associated eye diseases, general and visual outcome in smoking scleritis patients were similar to the nonsmoking patients in this study.

At present, we are not able to determine whether those who smoke should be treated more aggressively at presentation or whether simply stopping them from smoking during the course of treatment will allow the medication to retain its effectiveness. However, as a prospective trial is not feasible in a condition as uncommon as this we would feel on the evidence presented here that smoking should be prohibited during the treatment of these conditions.

\section{References}

1 Watson PG, Hayreh SS. Scleritis and episcleritis. Br J Ophthalmol 1976; 60: 163-191.

2 Tuft SJ, Watson PG. Progression of scleral disease. Ophthalmology 1991; 98: 467-471.

3 Watson P. In: Tasman W, Jaeger EA (eds). Diseases of the sclera and episclera. Duane's Clinical Ophthalmology. Chapter 23. Lippincott Williams \& Wilkins Publishers: Philadelphia, PA, 2002.

4 Schein JR. Cigarette smoking and clinically significant drug interactions. Ann Pharmacother 1995; 29: 1139-1148.

5 Yearly report STIVORO 2002. Look at: www.stivoro.nl., 2002.

6 Criswell LA, Merlino LA, Cerhan JR, Mikuls TR, Mudano AS, Burma M. Cigarette smoking and the risk of rheumatoid arthritis among postmenopausal women: results from the Iowa Women's Health Study. Am J Med 2002; 112: 465-471.

7 Ghadirian P, Dadgostar B, Azani R, Maisonneuve P. A case-control study of the association between sociodemographic, lifestyle and medical history factors and multiple sclerosis. Can J Public Health 2001; 92: 281-285.

8 Murin S, Bilello KS, Matthay R. Other smoking-affected pulmonary diseases. Clin Chest Med 2000; 21: 121-137,ix.

9 Bridger S, Lee JC, Bjarnason I, Jones JE, Macpherson AJ. In siblings with similar genetic susceptibility for inflammatory bowel disease, smokers tend to develop Crohn's disease and non-smokers develop ulcerative colitis. Gut 2002; 51: 21-25.

10 Bartalena L, Martino E, Marcocci C, Bogazzi F, Panicucci M, Velluzzi F. More on smoking habits and Graves' ophthalmopathy. J Endocrinol Invest 1989; 12: 733-737.

11 Prummel MF, Wiersinga WM. Smoking and risk of Graves' disease. JAMA 1993; 269: 479-482.

12 Wiersinga WM, Prummel MF. Graves' ophthalmopathy: a rational approach to treatment. Trends Endocrinol Metab 2002; 13: 280-287.

13 Bartalena L, Marcocci C, Tanda ML, Manetti L, Dell'Unto E, Bartolomei MP. Cigarette smoking and treatment outcomes in Graves ophthalmopathy. Ann Intern Med 1998; 129: 632-635.

14 Wiersinga WM, Prummel MF. An evidence-based approach to the treatment of Graves' ophthalmopathy. Endocrinol Metab Clin North Am 2000; 29: 297-319,vi-vii.

15 Nieuwenhuizen J, Watson PG, Jager MJ, Emmanouilidisvan der Spek K, Keunen JE. The value of combining anterior segment fluorescein angiography with indocyanine green angiography in scleral inflammation. Ophthalmology 2003; 110: $1653-1666$.

16 Grygiel JJ, Birkett DJ. Cigarette smoking and theophylline clearance and metabolism. Clin Pharmacol Ther 1981; 30: 491-496.

17 Nemeroff CB, DeVane CL, Pollock BG. Newer antidepressants and the cytochrome P450 system. Am J Psychiatry 1996; 153: 311-320.

18 Spigset O, Carleborg L, Hedenmalm K, Dahlqvist R. Effect of cigarette smoking on fluvoxamine pharmacokinetics in humans. Clin Pharmacol Ther 1995; 58: 399-403. 
19 Vinarova E, Vinar O, Kalvach Z. Smokers need higher doses of neuroleptic drugs. Biol Psychiatry 1984; 19: 1265-1268.

20 Goff DC, Henderson DC, Amico E. Cigarette smoking in schizophrenia: relationship to psychopathology and medication side effects. Am J Psychiatry 1992; 149: 1189-1194.

21 Hansten PD, Horn JR, Koda-Kimble MA, Young LYE. Effects of Cigarette Smoking on Drug Actions. Applied Therapeutics Inc.: Vancouver, 1993.

22 Vestal RE, Wood AJ. Influence of age and smoking on drug kinetics in man: studies using model compounds. Clin Pharmacokinet 1980; 5: 309-319.

23 Madsbad S, McNair P, Christensen MS, Christiansen C, Faber OK, Binder C. Influence of smoking on insulin requirement and metabolic status in diabetes mellitus. Diabetes Care 1980; 3: 41-43.

24 Clinical depression of the central nervous system due to diazepam and chlordiazepoxide in relation to cigarette smoking and age. N Engl J Med 1973; 288: 277-280.

25 Miller RR. Effects of smoking on drug action. Clin Pharmacol Ther 1977; 22: 749-756.

26 Dollery C, Brennan PJ. The Medical Research Council Hypertension Trial: the smoking patient. Am Heart J 1988; 115: 276-281.

27 Deanfield J, Wright C, Krikler S, Ribeiro P, Fox K. Cigarette smoking and the treatment of angina with propranolol, atenolol, and nifedipine. N Engl J Med 1984; 310: 951-954.
28 Holt PG. Immune and inflammatory function in cigarette smokers. Thorax 1987; 42: 241-249.

29 Holt PG, Keast D. Environmentally induced changes in immunological function: acute and chronic effects of inhalation of tobacco smoke and other atmospheric contaminants in man and experimental animals. Bacteriol Rev 1977; 41: 205-216.

30 Sopori M. Effects of cigarette smoke on the immune system. Nat Rev Immunol 2002; 2: 372-377.

31 Barbour SE, Nakashima K, Zhang JB, Tangada S, Hahn CL, Schenkein HA. Tobacco and smoking: environmental factors that modify the host response (immune system) and have an impact on periodontal health. Crit Rev Oral Biol Med 1997; 8: 437-460.

32 Albano SA, Santana-Sahagun E, Weisman MH. Cigarette smoking and rheumatoid arthritis. Semin Arthritis Rheum 2001; 31: 146-159.

33 Soy M, Erken E, Konca K, Ozbek S. Smoking and Behcet's disease. Clin Rheumatol 2000; 19: 508-509.

34 Mattoli S, Kleimberg J, Stacey MA, Bellini A, Sun G, Marini M. The role of CD8 + Th2 lymphocytes in the development of smoking-related lung damage. Biochem Biophys Res Commun 1997; 239: 146-149.

35 Reckner Olsson A, Skogh T, Wingren G. Comorbidity and lifestyle, reproductive factors, and environmental exposures associated with rheumatoid arthritis. Ann Rheum Dis 2001; 60: 934-939. 